\title{
ĐÁNH GIÁ KIỂM TRA THẾ TRỌNG TRƯỜNG W CỦA MẠTT GEOID CỤC Bộ HÒN DẤU TRÊN CƠ SỞ SỬ DỤNG 89 ĐIỂM ĐỘ CAO HẠNG I
}

\author{
PGS. TSKH. HÀ MINH HOÀ(1), ThS. NGUYẼN TH! THANH HƯO'NG ${ }^{(1)}$ \\ ThS. LU'O'NG THANH THACH ${ }^{(2)}$ \\ (1) Viện Khoa học Đo đạc và Bản đồ \\ ${ }^{(2)}$ Trường Đại học Tài nguyên và Môi trường Hà Nội
}

\section{Tóm tắt:}

Bài báo khoa học này đã tiến hành tính toán kiểm tra giá trị thế trọng trường $W_{0}=$ $62636847.291 \mathrm{~m}^{2} . \mathrm{s}^{-2}$ và giá trị độ cao $H_{0}=0.890 \mathrm{~m}$ của mặt geoid cục bộ Hòn Dấu so với mặt geoid toàn cầu dựa trên 89 điểm độ cao hạng I và mô hình địa hình động lực trung bình DTU10MDT quốc tế. Các kết quả kiểm tra một lần nữa xác định sự tin cậy của các giá trị nêu trên và làm cơ sở để sử dụng các giá trị này trong việc giải quyết các bài toán hiện đại của trắc địa vật lý ở nước ta.

\section{1. Đặt vấn đề}

Thế trọng trường $=62636856.00 \mathrm{~m}^{2} . \mathrm{s}^{-2}$ của mặt geoid toàn cầu sát nhất với mặt biển trung bình trên các biển và các đại dương thế giới được xác định nhờ các dữ liệu altimetry và được công bố trong tài liệu (Bursa M., Kenyon S., Kouba J., Radj K., Vatrt V., Vojtiskov., Simek J. (2002); Bursa M., Kenyon S., Kouba J., Sima Z., Vatrt V., Vitek V. and Vojtiskova M. (2007)). Giá trị thế trọng trường này của mặt geoid toàn cầu đã được Tổ chức Dịch vụ quay Trái đất quốc tế IERS (International Earth Rotation Service) công nhận trong các Hiệp ước (Conventions) 2004 và 2010 (Dennis D. McCarthy, Gerard Petit. (2004); Petit G., Luzum B. (2010)) và được sử dụng để xây dựng mô hình trọng trường Trái đất EGM2008 (M., Kenyon S., Kouba J., Sima Z., Vatrt V., Vitek V. and Vojtiskova M. (2007)).

Đối với mọi điểm M bất kỳ trên bề mặt Trái đất, dựa trên quan hệ (Hà Minh Hòa (2007)):

$$
\bar{H}_{M}^{\gamma}=H_{M}^{\gamma}+\frac{\bar{W}_{0}-W_{0}}{\bar{\gamma}_{M}}
$$

Ở đây $\bar{H}_{M}^{\gamma}$ - độ cao chuẩn toàn cầu của điểm $\mathrm{M}$ đượC xác định trong hệ triều 0 và tương ứng với mặt geoid toàn cầu, thêm vào đó $\bar{H}_{M}^{\gamma}=\bar{H}_{M}-\bar{\zeta}_{M}$, còn $\bar{H}_{M}$ - độ cao trắc địa của điểm $\mathrm{M}$ được xác định từ các kết quả xử lý các dữ liệu GPS trong ITRF tương ứng với ellipsoid WGS84 quốc tế và được chuyển về hệ triều $0, \bar{\zeta}_{M}$ - dị thường độ cao toàn cầu của điểm $\mathrm{M}$ được xác định từ mô hình EGM2008 và được chuyển về hệ triều $0 ; H_{M}^{\gamma}$ - độ cao chuẩn cục bộ của điểm $\mathrm{M}$ trong hệ độ cao quốc gia (tương ứng với mặt geoid cục bộ Hòn Dấu với thế trọng trường $\mathrm{W}_{0}$ ), khi coi các hiệu $\bar{H}^{\gamma}-H^{\gamma}$ trên các điểm độ cao hạng $\mathrm{I}$ quốc gia là các trị đo và kiểm tra sự có mặt của các sai số thô theo tiêu chuẩn Smirnov 
N.V. (Smirnov N.V., Belugin D.A. (1969)), trong hàng loạt các tài liệu, ví dụ Hà Minh Hòa, Nguyễn Thị Thanh Hương và nnk (2012), Ha Minh Hoa (2013), Ha Minh Hoa (2014), đã sử dụng 35 điểm độ cao hạng I phân bố đồng đều trên lãnh thổ Việt Nam để xác định thế trọng trường $W_{0}$ của mặt geoid cục bộ Hòn Dấu sát nhất với mặt biển trung bình nhiều năm tại trạm nghiệm triều Hòn Dấu theo phương pháp bình phương nhỏ nhất. Kết quả nhận được thế trọng trường $W_{0}=62636847.291 \mathrm{~m}^{2} \cdot \mathrm{s}^{-2} \pm 0.183 \mathrm{~m}^{2} \cdot \mathrm{s}^{-2}$ của mặt geoid cục bộ Hòn Dấu.

Đại lượng

$$
H_{0}=\frac{\bar{W}_{0}-W_{0}}{\bar{\gamma}_{M}}=\frac{8.7089 \mathrm{~m}^{2} \cdot \mathrm{s}^{-2}}{\bar{\gamma}_{M}},
$$

là độ cao của mặt geoid cục bộ Hòn Dấu so với mặt geoid toàn cầu, ở đây giá trị trung bình của gia tốc lực trọng trường chuẩn $\bar{\gamma}_{M}$ (đơn vị $\mathrm{m}^{-\mathrm{s}^{-2}}$ ) được xác định theo công thức:

$$
\bar{\gamma}_{M}=\gamma_{0}-10^{-5} \cdot\left(0.1543 \cdot H_{M}^{\gamma}+0.036 \cdot 10^{-6} \cdot\left(H_{M}^{\gamma}\right)^{2}\right) \text {, }
$$

còn giá trị $\gamma_{o}$ (đơn vị m.s.2) đối với ellipsoid WGS84 được xác định theo công thức:

$$
\gamma_{0}=9.7803253359 .\left(1+0.00530248 \cdot \sin ^{2} B-0.0000058497 . \sin ^{2} 2 B\right)<m \cdot s^{-2}>
$$

Ưu điểm cơ bản của việc xác định độ cao $\mathrm{H}_{0}(2)$ với $\bar{\gamma}_{M}$ (đơn vị $\mathrm{m}^{2}$. $^{-2}$ ) được xác định theo công thức (3) nằm ở chỗ các giá trị $\mathrm{H}_{0}$ được tính toán không bị ảnh hưởng của sự xê dịch của điểm $\mathrm{M}$ trên mặt vật lý Trái đất do các tác nhân tự nhiên (chuyển dịch đứng của vỏ Trái đất, sạt lở đất .v.v...) và các tác nhân nhân sinh (xê dịch mốc do mở rộng các đường giao thông, xây dựng các khu công nghiệp và đô thị .v.v...). Số chữ số có nghĩa của giá trị $\bar{\gamma}_{M}(3)$ để tính đại lượng $\mathrm{H}_{0}(2)$ chỉ là 3 chữ số sau dấu phẩy. Nếu độ cao chuẩn $H_{M}^{\gamma}$ của điểm $\mathrm{M}$ thay đổi đi $10 \mathrm{~m}$, thì điều này cũng chỉ ảnh hưởng đến số lẻ thứ 5 sau dấu phẩy của giá trị $\bar{\gamma}_{M}(3)$.

Trong các tài liệu (Hà Minh Hòa, Nguyễn Thị Thanh Hương và nnk (2012); Ha Minh Hoa (2013); Hà Minh Hòa (2014)) dựa trên 11 điểm trọng lực cơ sở và 29 điểm trọng lực hạng I phủ trùm cả nước đã xác định được rằng độ cao $\mathrm{H}_{0}=0.890 \mathrm{~m}$ và là đại lượng không đổi trên toàn bộ lãnh thổ Việt Nam. Kết quả tương tự cũng nhận được trong tài liệu (Nguyễn Tuấn Anh (2015)) khi sử dụng 133 điểm trọng lực chi tiết được phân bố trên 09 vùng Tây Bắc, Đông Bắc, Tây Tây Bắc, Đông Đông Bắc, Bắc Trung Bộ, Trung Bộ, Nam Trung Bộ, Tây Nguyên và Nam Bộ.

Do sự quan trọng của giá trị thế trọng trường $W_{0}=62636847.2911 \mathrm{~m}^{2} \cdot \mathrm{s}^{-2} \pm 0.183 \mathrm{~m}^{2} \cdot \mathrm{s}^{-2}$ của mặt geoid cục bộ Hòn Dấu và độ cao $\mathrm{H}_{0}=0.890 \mathrm{~m}=$ const trong việc giải quyết các bài toán trắc địa vật lý, trong bài báo khoa học này sẽ giải quyết 02 vấn đề:

- Luận chứng cho độ cao $\mathrm{H}_{0}=0.890 \mathrm{~m}$ giữa mặt quasigeoid cục bộ Hòn Dấu và là đại lượng không đổi trên toàn bộ lãnh thổ Việt Nam;

- Đánh giá kiểm tra giá trị thế trọng trường $\mathrm{W}_{0}=62636847.291 \mathrm{~m}^{2} . \mathrm{s}^{-2} \pm 0.183 \mathrm{~m}^{2} \cdot \mathrm{s}^{-2}$ của mặt geoid cục bộ Hòn Dấu khi mở rộng số lượng các điểm độ cao hạng I quốc gia và sử dụng mô hình địa hình động lực trung bình DTU10 MDT. 


\section{Giải quyết vấn đề}

Chúng ta sẽ giải quyết vấn đề thứ nhất dựa trên hình 1 ở dưới đây. Đối với điểm $\mathrm{M}$ bất kỳ trên bề mặt Trái đất, điểm $P$ là hình chiếu của điểm $M$ lên mặt quasigeoid cục bộ Hòn Dấu, điểm $\mathrm{Q}$ là hình chiếu của điểm $\mathrm{M}$ lên mặt quasigeoid toàn cầu theo đường vuông góc với mặt ellipsoid. Lưu ý rằng mặt quasigeoid cục bộ Hòn Dấu và mặt quasigeoid toàn cầu được xác định trong trọng trường chuẩn của ellipsoid, từ các chứng minh trong tài liệu $(\mathrm{Ha}$ Minh Hoa (2014)) chúng ta xác định được các thế chuẩn của các điểm $\mathrm{P}, \mathrm{Q}$ theo công thức sau:

$$
\begin{aligned}
& U_{P}=W_{0}-T_{M}, \\
& U_{Q}=\overline{W_{0}}-T_{M},
\end{aligned}
$$

ở đây $T_{M}$ là thế nhiễu tại điểm $M$.

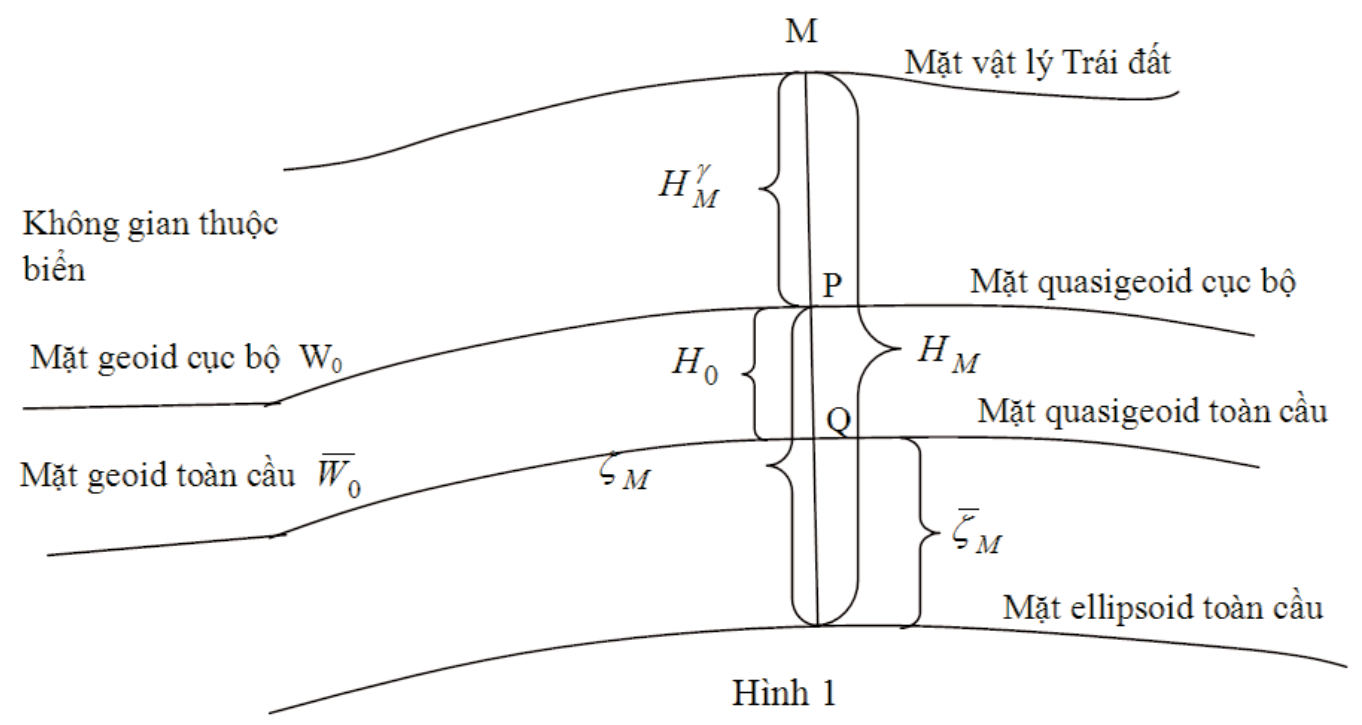

Từ công thức (4) lưu ý (2) chúng ta nhận được công thức:

$$
H_{0}=\frac{\bar{W}_{0}-W_{0}}{\bar{\gamma}_{M}}=\frac{U_{Q}-U_{P}}{\bar{\gamma}_{M}}=\frac{8.7089 \mathrm{~m}^{2} \cdot \mathrm{s}^{-2}}{\bar{\gamma}_{M}} .
$$

Do $\frac{U_{Q}-U_{P}}{\bar{\gamma}_{M}}$ là độ cao của mặt quasigeoid cục bộ Hòn Dấu so với mặt quasigeoid toàn cầu tương ứng với điểm $\mathrm{M}$, nên từ (5) chúng ta thấy rằng hiệu thế trọng trường $\bar{W}_{0}-W_{0}=8,7089 \mathrm{~m}^{2} \cdot \mathrm{s}^{-2}$ được xác định tại trạm nghiệm triều Hòn Dấu và được kế thừa để tính toán độ cao của mặt quasigeoid cục bộ Hòn Dấu so với mặt quasigeoid toàn cầu tương ứng với điểm $\mathrm{M}$ bất kỳ trên toàn bộ lãnh thổ Việt Nam. Các kết quả nghiên cứu cho thấy trên toàn bộ lãnh thổ Việt Nam, giá trị nhỏ nhất $\bar{\gamma}_{\min }=9.782 \mathrm{~ms}^{-2}$, giá trị lớn nhất $\bar{\gamma}_{\max }=9.788 \mathrm{mss}^{-2}$ và hiệu $\bar{\gamma}_{\max }-\bar{\gamma}_{\min }=0.006 \mathrm{mss}^{-2}=600 \mathrm{mGal}$.

Do 


$$
\begin{aligned}
& H_{0}=\frac{8.7089 \mathrm{~m}^{2} \cdot \mathrm{s}^{-2}}{\bar{\gamma}_{\min }}=\frac{8.7089 \mathrm{~m}^{2} \cdot \mathrm{s}^{-2}}{9.782}=0.8902 \mathrm{~m}=0.890 \mathrm{~m}, \\
& H_{0}=\frac{8.7089 \mathrm{~m}^{2} \cdot \mathrm{s}^{-2}}{\bar{\gamma}_{\max }}=\frac{8.7089 \mathrm{~m}^{2} \cdot \mathrm{s}^{-2}}{9.788}=0.8897 \mathrm{~m}=0.890 \mathrm{~m},
\end{aligned}
$$

nên không khó khăn để xác nhận rằng trên toàn bộ lãnh thổ Việt $\mathrm{Nam}$, độ cao $\mathrm{H}_{0}=0.890$ $m$ giữa mặt quasigeoid cục bộ Hòn Dấu và là đại lượng không đổi. Tại đỉnh Fanxipan với độ cao $3143 \mathrm{~m}$ và các tọa độ trắc địa $\mathrm{B}=20^{\circ} 17^{\prime} 52^{\prime \prime}, \mathrm{L}=103^{\circ} 47^{\prime} 11^{\prime \prime}$, giá trị $\bar{\gamma}_{\text {phanxipan }}=9.78172{\mathrm{~m} . \mathrm{s}^{-2}}$,

$$
H_{0}=\frac{8.7089 \mathrm{~m}^{2} \cdot \mathrm{s}^{-2}}{9.78172 \mathrm{~ms}^{-2}}=0.89033 \mathrm{~m}=0.890 \mathrm{~m} .
$$

Bây giờ chúng ta sẽ giải quyết vấn đề thứ hai theo hai cách tiếp cận khác nhau. Đối với cách tiếp cận thứ nhất, chúng ta mở rộng các điểm độ cao hạng I tham gia tính toán thế trọng trường Hòn Dấu từ 35 điểm lên đến 89 điểm. Đối với cách tiếp cận thứ hai, chúng ta sẽ sử dụng mô hình địa hình động lực trung bình quốc tế DTU10MDT.

Đối với cách tiếp cận thứ nhất, chúng ta phải chuyển các giá trị độ cao chuẩn quốc gia của các điểm độ cao hạng I từ hệ triều trung bình về hệ triều 0 , chuyển các giá trị độ cao trắc địa (được xác định từ kết quả xử lý các dữ liệu đo GPS trên các điểm độ cao hạng I trong ITRF tương ứng với ellipsoid WGS84 quốc tế) và các giá trị dị thường độ cao toàn cầu (được xác định từ mô hình trọng trường Trái đất EGM2008) từ hệ không phụ thuộc triều về hệ triều 0 . Trên mỗi điểm độ cao hạng I thứ $\mathrm{i}(\mathrm{i}=1,2, \ldots, \mathrm{n})$, chúng ta xác định hiệu $\bar{H}_{i}^{\gamma}-H_{i}^{\gamma}$, ở đây $\bar{H}_{i}^{\gamma}=\bar{H}_{i}-\bar{\zeta}_{i}$ - độ cao chuẩn toàn cầu của điểm độ cao hạng I thứ i. Trên 89 điểm độ cao hạng I quốc gia đã xác định được các giá trị độ cao trắc địa $\bar{H}$ và các giá trị dị thường độ cao toàn cầu $\zeta$ và đã tạo được dãy các đại lượng $\bar{H}_{i}^{\gamma}-H_{i}^{\gamma}$ (xem cột $\mathrm{g}$ trong bảng 1).

Trên điểm độ cao hạng I thứ i $(\mathrm{i}=1,2, \ldots, \mathrm{n})$, khi coi $y_{i}=\bar{\gamma}_{i} \cdot\left(\bar{H}_{i}^{\gamma}-H_{i}^{\gamma}\right)$ là trị đo, từ công thức (1) chúng ta lập được phương trình số cải chính của trị đo $y_{i}$ ở dạng sau $v_{i}=-W_{0}+\left(\bar{W}_{0}-y_{i}\right)$ với ẩn số cần tìm là thế trọng trường $W_{0}$ của mặt geoid cục bộ Hòn Dấu sát nhất với mặt biển trung bình tại trạm nghiệm triều Hòn Dấu, ở đây $\bar{W}_{0}=$ $62636856.00 \mathrm{~m}^{2} . \mathrm{s}^{-2}$ - thế trọng trường của mặt geoid toàn cầu. Đối với $\mathrm{n}$ trị đo độc lập $y_{i}$ ( $\mathrm{i}=1,2, \ldots, \mathrm{n}$ ) trên $\mathrm{n}$ điểm độ cao hạng $\mathrm{I}$, giải hệ phương trình số cải chính nếu trên dưới điều kiện $\sum_{i=1}^{n} v_{i}^{2}=\min$, chúng ta nhận được phương trình chuẩn $-W_{0}+\bar{W}_{0}-\frac{1}{n} \cdot \sum_{i=1}^{n} y_{i}=0$ hay

$$
W_{0}=\bar{W}_{0}-\bar{y}=62636856.00 \mathrm{~m}^{2} \cdot \mathrm{s}^{-2}-\bar{y},
$$

ở đây $\bar{y}=\frac{1}{n} \cdot \sum_{i=1}^{n} y_{i}$ - giá trị trung bình của các trị đo $y_{i}(\mathrm{i}=1,2, \ldots, \mathrm{n})$ trên $\mathrm{n}$ điểm độ cao hạng I. 
Tuy nhiên, với mục đích đánh giá thế trọng trường $W_{0}$ của mặt geoid cục bộ Hòn Dấu theo công thức $(6)$, chúng ta phải đảm bảo rằng trong các trị đo $y_{i}(\mathrm{i}=1,2, \ldots, \mathrm{n})$ không chứa các sai số thô. Chúng ta sẽ tiến hành kiểm tra sự có mặt của các sai số thô trong các trị đo $y_{i}$ theo tiêu chuẩn Smirnov. Giả thiết rằng trong một nhóm gồm $\mathrm{m}$ các trị đo $y_{i}(\mathrm{i}=$ $1,2, \ldots, \mathrm{m})$, chúng ta phải sắp xếp các trị đo theo thứ tự tăng dần theo giá trị tuyệt đối của chúng, tính giá trị trung bình $\bar{y}_{m}=\frac{1}{m} \cdot \sum_{i=1}^{m} y_{i}$ và các độ lệch $\delta_{i}=y_{i}-\bar{y}_{m} \quad(\mathrm{i}=1,2, \ldots, \mathrm{m})$. Tiếp theo chúng ta xác định sai số trung phương $S= \pm \mid \frac{\sum_{i=1}^{m} \delta_{i}^{2}}{m}$. Đối với trị đo $y_{1}$ có giá trị tuyệt đối nhỏ nhất nằm ở vị trị đầu tiên chúng ta xác định giá trị $r_{\min }=\left|\frac{\delta_{1}}{S}\right|$, còn đối với trị đo $y_{m}$ có giá trị tuyệt đối lớn nhất nằm ở vị trị cuối cùng trong nhóm chúng ta xác định giá trị $r_{\max }=\left|\frac{\delta_{m}}{S}\right|$. Với mức giá trị $5 \%$, dựa vào Bảng các giá trị của tiêu chuẩn Smirnov (xem Phụ lục 3 trong tài liệu Hà Minh Hòa (2014)) chúng ta xác định giá trị $\bar{F}_{*}$ Nếu $\bar{r}>_{r_{\min }}$ và $\bar{r}>_{r_{\max }}$, thì các trị đo $y_{i}(\mathrm{i}=1,2, \ldots, \mathrm{m})$ trong nhóm các trị đo đang nghiên cứu không chứa các sai số thô.

Do trong Bảng các giá trị của tiêu chuẩn Smirnov chỉ cho giá trị pới với lượng $m$ tối đa bằng 40 , nên từ 89 các trị đo $y_{i}$ trong bảng 2 chúng ta phải chia thành 03 nhóm các trị đo, các nhóm trị đo thứ nhất và thứ hai đều có 40 các trị đo, riêng nhóm các trị đo thứ ba chỉ có 09 trị đo. Các kết quả kiểm tra sự có mặt của các sai số thô trong 03 dãy nhỏ được trình bày trong các bảng $3,4,5$.

\section{Bảng 1}

\begin{tabular}{|c|c|c|c|c|c|c|c|}
\hline $\begin{array}{c}\text { STT } \\
\mathrm{i}\end{array}$ & Tên điểm & \begin{tabular}{|} 
Độ cao \\
chuẩn quốc \\
gia $H_{i}^{\gamma}$ \\
trong hệ triëu \\
$0(\mathrm{~m})$
\end{tabular} & 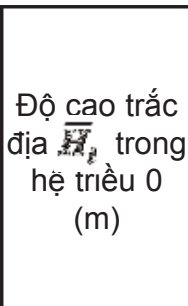 & $\begin{array}{c}\text { Dị thường độ } \\
\text { cao trọng lực } \\
\overline{\bar{F}_{i}} \text { từ } \\
\text { EGM2008 } \\
\text { trong hệ triều } \\
0(\mathrm{~m})\end{array}$ & $\begin{array}{c}\text { Độ cao chuần } \\
\text { toàn cầu } \bar{H}_{i}^{\gamma} \\
\text { so với mặt } \\
\text { geoid toàn cầu } \\
\text { trong hệ triều } 0 \\
\text { (m) }\end{array}$ & $\begin{array}{c}\text { Hiệu } \\
\bar{H}_{i}^{\gamma}-H_{i}^{\gamma} \\
\quad(\mathrm{m})\end{array}$ & $\begin{array}{c}\text { Giá trị trung } \\
\text { bình của gia } \\
\text { tốc lực trọng } \\
\text { trường } \\
\text { chuẩn } \\
\overline{y_{y}}\left(\mathrm{~m}^{-\mathrm{s}^{-2}}\right)\end{array}$ \\
\hline a & $\mathrm{b}$ & c & d & e & $f$ & $\mathrm{~g}$ & $\mathrm{~h}$ \\
\hline 1 & I(BH-LS)97 & 280,727 & 255.349 & -26.361 & 281.710 & 0.983 & 9.787117 \\
\hline 2 & $\mathrm{I}(\mathrm{BH}-\mathrm{TH}) 122 \mathrm{~A}$ & 239,838 & 212.958 & -27.776 & 240.734 & 0.870 & 9.786434 \\
\hline 3 & $\mathrm{I}(\mathrm{BH}-\mathrm{TH}) 119$ & 377,631 & 350.744 & -27.803 & 378.547 & 0.916 & 9.786821 \\
\hline 4 & $\mathrm{I}(\mathrm{BH}-\mathrm{HN}) 33$ & 13,102 & -15.210 & -29.211 & 14.001 & 0.899 & 9.787156 \\
\hline 5 & $\mathrm{I}(\mathrm{BH}-\mathrm{HN}) 39$ & 11,385 & -16.613 & -28.902 & 12.289 & 0.904 & 9.787148 \\
\hline 6 & $\mathrm{I}(\mathrm{BH}-\mathrm{HN}) 42$ & 7,558 & -20.214 & -28.648 & 8.434 & 0.876 & 9.787120 \\
\hline 7 & $\mathrm{I}(\mathrm{HN}-\mathrm{HP}) 7$ & 3,046 & -23.594 & -27.589 & 3.995 & 0.949 & 9.786935 \\
\hline 8 & $\mathrm{I}(\mathrm{HN}-\mathrm{VL}) 10 \mathrm{~A}$ & 4,764 & -21.544 & -27.105 & 5.561 & 0.797 & 9.786707 \\
\hline
\end{tabular}


Nghiên cứu

\begin{tabular}{|c|c|c|c|c|c|c|c|}
\hline 9 & $\mathrm{I}(\mathrm{HN}-\mathrm{VL}) 4-1$ & 3,956 & -22.999 & -27.868 & 4.869 & 0.913 & 9.786885 \\
\hline 10 & $\mathrm{I}(\mathrm{HN}-\mathrm{VL}) 6-1$ & 3,066 & -23.625 & -27.575 & 3.950 & 0.884 & 9.786815 \\
\hline 11 & I(DN-BT)16 & 54.671 & 45.971 & -9.493 & 55.464 & 0.793 & 9.784017 \\
\hline 12 & I(DN-BT)28 & 559,764 & 552.371 & -8.192 & 560.563 & 0.799 & 9.783076 \\
\hline 13 & I(VL-HT)150 & 2,663 & 6.344 & 2.886 & 3.458 & 0.795 & 9.782820 \\
\hline 14 & $\mathrm{I}(\mathrm{VL}-\mathrm{HT}) 152-1$ & 10,235 & 14.461 & 3.382 & 11.079 & 0.844 & 9.782778 \\
\hline 15 & $\mathrm{I}(\mathrm{HN}-\mathrm{VL}) 34-1$ & 3,818 & -20.399 & -25.035 & 4.636 & 0.818 & 9.786054 \\
\hline 16 & I(HP-MC)48A & 6,821 & -15.323 & -22.966 & 7.643 & 0.822 & 9.787301 \\
\hline 17 & $\mathrm{I}(\mathrm{BH}-\mathrm{TH}) 3-1$ & 73,286 & 42.526 & -31.606 & 0.846 & 0.845 & 9.787678 \\
\hline 18 & I(VL-HT)181 & 20,380 & 25.947 & 4.761 & 21.186 & 0.806 & 9.782444 \\
\hline 19 & I(LS-TY)4 & 298.250 & 274.012 & -25.068 & 299.080 & 0.830 & 9.786933 \\
\hline 20 & $\mathrm{I}(\mathrm{VL}-\mathrm{HT}) 309 \mathrm{~A}$ & 0.701 & -6.786 & -8.296 & 1.510 & 0.809 & 9.781873 \\
\hline 21 & I(VL-HT)317 & 0.880 & -7.996 & -9.690 & 1.694 & 0.814 & 9.781944 \\
\hline 22 & $\mathrm{I}(\mathrm{VL}-\mathrm{HT}) 187$ & 11.226 & 16.532 & 4.488 & 12.054 & 0.828 & 9.782377 \\
\hline 23 & $\mathrm{I}(\mathrm{VL}-\mathrm{HT}) 170-1$ & 6.658 & 11.501 & 4.024 & 7.487 & 0.829 & 9.782598 \\
\hline 24 & I(HP-MC)41 & 5.729 & -16.709 & -23.286 & 15.577 & 0.848 & 9.787258 \\
\hline 25 & I(VL-HT)130 & 7.991 & 10.001 & 1.178 & 8.823 & 0.832 & 9.783027 \\
\hline 26 & $\mathrm{I}(\mathrm{HN}-\mathrm{VL}) 56$ & 11.200 & -11.852 & -23.970 & 12.118 & 0.918 & 9.785546 \\
\hline 27 & $\mathrm{I}(\mathrm{BH}-\mathrm{TH}) 11$ & 372.392 & 341.376 & -31.847 & 373.223 & 0.931 & 9.787309 \\
\hline 28 & $\mathrm{I}(\mathrm{HN}-\mathrm{VL}) 40-1$ & 2.768 & -21.309 & -25.001 & 3.692 & 0.924 & 9.785891 \\
\hline 29 & $\mathrm{I}(\mathrm{BH}-\mathrm{LS}) 77$ & 207.379 & 180.239 & -28.073 & 208.312 & 0.933 & 9.787665 \\
\hline 30 & $\mathrm{I}(\mathrm{BH}-\mathrm{TH}) 5$ & 76.874 & 45.917 & -31.809 & 77.726 & 0.852 & 9.787723 \\
\hline 31 & I(HN-VL)38-1 & 3.647 & -20.504 & -24.999 & 4.495 & 0.848 & 9.785949 \\
\hline 32 & I(VL-HT)197 & 16.897 & 21.844 & 4.112 & 17.732 & 0.835 & 9.782266 \\
\hline 33 & I(BT-APD)63 & 9.819 & 6.590 & -4.064 & 10.654 & 0.835 & 9.782192 \\
\hline 34 & $\mathrm{I}(\mathrm{VL}-\mathrm{HT}) 127-3$ & 2.796 & 4.147 & 0.510 & 3.637 & 0.841 & 9.783113 \\
\hline 35 & I(BT-APD)59-1 & 18.073 & 14.316 & -4.595 & 18.911 & 0.838 & 9.782230 \\
\hline 36 & $\mathrm{I}(\mathrm{VL}-\mathrm{HT}) 278-1$ & 1.688 & -2.619 & -5.151 & 2.532 & 0.844 & 9.782015 \\
\hline 37 & $\mathrm{I}(\mathrm{VL}-\mathrm{HT}) 108$ & 23.780 & 22.232 & -2.400 & 24.632 & 0.852 & 9.783400 \\
\hline 38 & I(DN-BT)77 & 647.161 & 644.437 & -3.579 & 648.016 & 0.855 & 9.782261 \\
\hline 39 & I(BT-NH)17-1 & 421.022 & 423.816 & 1.942 & 421.874 & 0.852 & 9.782845 \\
\hline 40 & $\mathrm{I}(\mathrm{VL}-\mathrm{HT}) 83$ & 5.707 & 0.511 & -6.054 & 6.665 & 0.958 & 9.783842 \\
\hline 41 & $\mathrm{I}(\mathrm{BH}-\mathrm{HN}) 17$ & 32.017 & 2.783 & -30.107 & 32.890 & 0.873 & 9.787365 \\
\hline 42 & $\mathrm{I}(\mathrm{HN}-\mathrm{VL}) 45-1$ & 3.230 & -20.284 & -24.434 & 4.150 & 0.920 & 9.785774 \\
\hline
\end{tabular}


Nghiên cứu

\begin{tabular}{|c|c|c|c|c|c|c|c|}
\hline 43 & $\mathrm{I}(\mathrm{BH}-\mathrm{TH}) 65$ & 343.222 & 311.806 & -32.298 & 344.104 & 0.882 & 9.786915 \\
\hline 44 & $\mathrm{I}(\mathrm{VL}-\mathrm{HT}) 178$ & 2.810 & 8.146 & 4.468 & 3.678 & 0.868 & 9.782512 \\
\hline 45 & $\mathrm{I}(\mathrm{VL}-\mathrm{HT}) 103$ & 7.363 & 5.245 & -2.993 & 8.238 & 0.875 & 9.783515 \\
\hline 46 & $\mathrm{I}(\mathrm{HN}-\mathrm{VL}) 64$ & 2.377 & -19.430 & -22.691 & 3.261 & 0.884 & 9.785400 \\
\hline 47 & $\mathrm{I}(\mathrm{VL}-\mathrm{HT}) 141-3$ & 150.480 & 154.017 & 2.661 & 151.356 & 0.876 & 9.782666 \\
\hline 48 & I(VL-HT)329A & 0.818 & -10.143 & -11.837 & 1.694 & 0.876 & 9.782011 \\
\hline 49 & $\mathrm{I}(\mathrm{HN}-\mathrm{VL}) 72$ & 8.265 & -12.452 & -21.608 & 9.156 & 0.891 & 9.785291 \\
\hline 50 & $\mathrm{I}(\mathrm{VL}-\mathrm{HT}) 158$ & 3.291 & 7.578 & 3.397 & 4.181 & 0.890 & 9.782711 \\
\hline 51 & $\mathrm{I}(\mathrm{VL}-\mathrm{HT}) 121$ & 8.074 & 8.536 & -0.487 & 9.023 & 0.949 & 9.783248 \\
\hline 52 & $\mathrm{I}(\mathrm{DN}-\mathrm{BT}) 74$ & 813.595 & 810.662 & -3.845 & 814.507 & 0.912 & 9.782069 \\
\hline 53 & I(BH-LS)88-1 & 185.119 & 158.731 & -27.302 & 186.033 & 0.914 & 9.787457 \\
\hline 54 & $\mathrm{I}(\mathrm{VL}-\mathrm{HT}) 98$ & 1.001 & -1.714 & -3.614 & 1.900 & 0.899 & 9.783604 \\
\hline 55 & $\mathrm{I}(\mathrm{BH}-\mathrm{LS}) 85-1$ & 174.898 & 148.363 & -27.453 & 175.816 & 0.918 & 9.787516 \\
\hline 56 & I(BH-LS)93 & 173.592 & 147.532 & -26.976 & 174.508 & 0.916 & 9.787391 \\
\hline 57 & $\mathrm{I}(\mathrm{BH}-\mathrm{LS}) 71$ & 359.091 & 331.541 & -28.471 & 360.012 & 0.921 & 9.787467 \\
\hline 58 & I(BT-APD)56 & 45.625 & 41.774 & -4.752 & 46.526 & 0.901 & 9.782237 \\
\hline 59 & $\mathrm{I}(\mathrm{VL}-\mathrm{HT}) 87$ & 3.626 & -0.735 & -5.279 & 4.544 & 0.918 & 9.783768 \\
\hline 60 & $\mathrm{I}(\mathrm{VL}-\mathrm{HT}) 247 \mathrm{~A}$ & 9.231 & 7.063 & -3.080 & 10.143 & 0.912 & 9.782179 \\
\hline 61 & I(LS-TY)1 & 269.367 & 244.166 & -26.133 & 270.299 & 0.932 & 9.787095 \\
\hline 62 & I(VL-HT)325-1 & 0.567 & -9.446 & -10.978 & 1.532 & 0.965 & 9.781980 \\
\hline 63 & I(DN-BT)83 & 385.901 & 383.600 & -3.220 & 386.820 & 0.919 & 9.782587 \\
\hline 64 & $\mathrm{I}(\mathrm{VL}-\mathrm{HT}) 78$ & 2.816 & -2.972 & -6.710 & 3.738 & 0.922 & 9.783928 \\
\hline 65 & $\mathrm{I}(\mathrm{LS}-\mathrm{HN}) 7$ & 121.183 & 95.916 & 26.212 & 122.128 & 0.945 & 9.787233 \\
\hline 66 & $\mathrm{I}(\mathrm{VL}-\mathrm{HT}) 71$ & 2.822 & -4.445 & -8.208 & 3.763 & 0.941 & 9.784046 \\
\hline 67 & $\mathrm{I}(\mathrm{BH}-\mathrm{TH}) 59$ & 374.104 & 342.469 & -32.599 & 375.068 & 0.964 & 9.786966 \\
\hline 68 & I(VL-HT)173-2 & 1.987 & 7.246 & 4.313 & 2.821 & 0.834 & 9.782561 \\
\hline 69 & $\mathrm{I}(\mathrm{BH}-\mathrm{TH}) 70 \mathrm{~A}$ & 563.648 & 532.639 & -31.974 & 564.613 & 0.965 & 9.786477 \\
\hline 70 & $\mathrm{I}(\mathrm{HN}-\mathrm{VL}) 50$ & 5.343 & -18.055 & -24.358 & 6.303 & 0.960 & 9.785640 \\
\hline 71 & I(VL-HT)123 & 232.772 & 233.249 & -0.477 & 233.726 & 0.954 & 9.782868 \\
\hline 72 & $\mathrm{I}(\mathrm{LS}-\mathrm{HN}) 12$ & 42.371 & 16.737 & -26.603 & 43.340 & 0.969 & 9.787282 \\
\hline 73 & $\mathrm{I}(\mathrm{HP}-\mathrm{MC}) 4-1$ & 1.562 & -23.100 & -25.637 & 2.537 & 0.975 & 9.786993 \\
\hline 74 & $\mathrm{I}(\mathrm{BH}-\mathrm{LS}) 80$ & 485.374 & 458.547 & -27.804 & 486.351 & 0.977 & 9.787167 \\
\hline 75 & I(DN-BT)86 & 374.348 & 372.264 & -3.043 & 375.307 & 0.959 & 9.782563 \\
\hline 76 & I(VL-HT)320A & 1.552 & -7.949 & -10.458 & 2.509 & 0.957 & 9.781970 \\
\hline
\end{tabular}


Nghiên cứu

\begin{tabular}{|c|c|c|c|c|c|c|c|}
\hline 77 & $\mathrm{I}(\mathrm{BT}-\mathrm{APD}) 49-1$ & 67.728 & 63.773 & -4.937 & 68.710 & 0.982 & 9.782261 \\
\hline 78 & $\mathrm{I}(\mathrm{HP}-\mathrm{NB}) 14 \mathrm{~A}$ & 0.868 & -24.765 & -26.401 & 1.636 & 0.768 & 9.786668 \\
\hline 79 & $\mathrm{I}(\mathrm{LS}-\mathrm{HN}) 36$ & 7.039 & -20.128 & -28.099 & 7.971 & 0.932 & 9.787032 \\
\hline 80 & $\mathrm{I}(\mathrm{LS}-\mathrm{HN}) 22$ & 12.674 & -14.025 & -27.472 & 13.447 & 0.773 & 9.787193 \\
\hline 81 & $\mathrm{I}(\mathrm{LS}-\mathrm{HN}) 29$ & 3.556 & -23.422 & -27.823 & 4.401 & 0.845 & 9.787135 \\
\hline 82 & $\mathrm{I}(\mathrm{BH}-\mathrm{HN}) 16 \mathrm{~A}$ & 32.460 & 3.731 & -29.692 & 33.423 & 0.963 & 9.787364 \\
\hline 83 & $\mathrm{I}(\mathrm{HN}-\mathrm{VL}) 28-1$ & 2.291 & -21.895 & -25.085 & 3.190 & 0.899 & 9.786258 \\
\hline 84 & $\mathrm{I}(\mathrm{BMT}-\mathrm{APD}) 30$ & 264.014 & 262.150 & -2.913 & 265.063 & 1.049 & 9.782091 \\
\hline 85 & $\mathrm{I}(\mathrm{BH}-\mathrm{HN}) 48$ & 7.974 & -19.340 & -28.327 & 8.987 & 1.013 & 9.786944 \\
\hline 86 & $\mathrm{I}(\mathrm{HN}-\mathrm{HP}) 2 \mathrm{~A}$ & 6.828 & -20.322 & -28.153 & 7.831 & 1.003 & 9.787012 \\
\hline 87 & $\mathrm{I}(\mathrm{HN}-\mathrm{HP}) 5$ & 3.938 & -22.897 & -27.872 & 4.975 & 1.037 & 9.786971 \\
\hline 88 & $\mathrm{I}(\mathrm{VL}-\mathrm{HT}) 73$ & 1.460 & -4.824 & -7.346 & 2.522 & 1.062 & 9.784012 \\
\hline 89 & $\mathrm{I}(\mathrm{VL}-\mathrm{HT}) 95$ & 4.303 & 23.422 & -3.778 & 5.347 & 1.044 & 9.783663 \\
\hline
\end{tabular}

Bảng 2

\begin{tabular}{|c|c|c|c|c|c|c|c|}
\hline $\begin{array}{l}\text { STT } \\
\mathrm{i}\end{array}$ & Tên điểm & $\begin{array}{c}\text { Trị đo } y_{i} \\
\left(\mathrm{~m}^{2} \cdot \mathrm{s}^{-2}\right)\end{array}$ & $\begin{array}{c}\Delta_{y} \\
\left(m^{2} \cdot s^{-2}\right)\end{array}$ & $\begin{array}{l}\text { STT } \\
\mathrm{i}\end{array}$ & Tên điểm & $\begin{array}{l}\text { Trị đo } y_{i} \\
\left(\mathrm{~m}^{2} \cdot \mathrm{s}^{-2}\right)\end{array}$ & $\frac{\Delta_{g}}{\left(m^{2} \cdot s^{-2}\right)}$ \\
\hline 1 & I(BH-LS)97 & 9.6207 & 0.9170 & 46 & $\mathrm{I}(\mathrm{HN}-\mathrm{VL}) 64$ & 8.6503 & -0.0534 \\
\hline 2 & $\mathrm{I}(\mathrm{BH}-\mathrm{TH}) 122 \mathrm{~A}$ & 8.5142 & -0.1895 & 47 & I(VL-HT)141-3 & 8.5696 & -0.1341 \\
\hline 3 & $\mathrm{I}(\mathrm{BH}-\mathrm{TH}) 119$ & 8.9647 & 0.2610 & 48 & I(VL-HT)329A & 8.5690 & -0.1347 \\
\hline 4 & $\mathrm{I}(\mathrm{BH}-\mathrm{HN}) 33$ & 8.7987 & 0.0950 & 49 & $\mathrm{I}(\mathrm{HN}-\mathrm{VL}) 72$ & 8.7187 & 0.0150 \\
\hline 5 & $\mathrm{I}(\mathrm{BH}-\mathrm{HN}) 39$ & 8.8476 & 0.1439 & 50 & $\mathrm{I}(\mathrm{VL}-\mathrm{HT}) 158$ & 8.7066 & 0.0029 \\
\hline 6 & $\mathrm{I}(\mathrm{BH}-\mathrm{HN}) 42$ & 8.5735 & 0.0498 & 51 & $\mathrm{I}(\mathrm{VL}-\mathrm{HT}) 121$ & 9.2843 & 0.5806 \\
\hline 7 & $\mathrm{I}(\mathrm{HN}-\mathrm{HP}) 7$ & 9.2878 & 0.5841 & 52 & I(DN-BT)74 & 8.9212 & 0.2175 \\
\hline 8 & $\mathrm{I}(\mathrm{HN}-\mathrm{VL}) 10 \mathrm{~A}$ & 7.8000 & -0.9037 & 53 & $\mathrm{I}(\mathrm{BH}-\mathrm{LS}) 88-1$ & 8.9457 & 0.2420 \\
\hline 9 & $\mathrm{I}(\mathrm{HN}-\mathrm{VL}) 4-1$ & 8.9354 & 0.2317 & 54 & $\mathrm{I}(\mathrm{VL}-\mathrm{HT}) 98$ & 8.7955 & 0.0918 \\
\hline 10 & $\mathrm{I}(\mathrm{HN}-\mathrm{VL}) 6-1$ & 8.6515 & -0.0522 & 55 & I(BH-LS)85-1 & 8.9849 & 0.2812 \\
\hline 11 & $\mathrm{I}(\mathrm{DN}-\mathrm{BT}) 16$ & 7.7587 & -0.9450 & 56 & I(BH-LS)93 & 8.9653 & 0.2616 \\
\hline 12 & I(DN-BT)28 & 7.8167 & -0.8870 & 57 & I(BH-LS)71 & 9.0143 & 0.3106 \\
\hline 13 & $\mathrm{I}(\mathrm{VL}-\mathrm{HT}) 150$ & 7.7773 & -0.9264 & 58 & I(BT-APD)56 & 8.8138 & 0.1101 \\
\hline 14 & $\mathrm{I}(\mathrm{VL}-\mathrm{HT}) 152-1$ & 8.2567 & -0.4470 & 59 & $\mathrm{I}(\mathrm{VL}-\mathrm{HT}) 87$ & 8.9815 & 0.2778 \\
\hline 15 & $\mathrm{I}(\mathrm{HN}-\mathrm{VL}) 34-1$ & 8.0050 & -0.6987 & 60 & $\mathrm{I}(\mathrm{VL}-\mathrm{HT}) 247 \mathrm{~A}$ & 8.9213 & 0.2176 \\
\hline 16 & $\mathrm{I}(\mathrm{HP}-\mathrm{MC}) 48 \mathrm{~A}$ & 8.0452 & -0.6585 & 61 & I(LS-TY)1 & 9.1216 & 0.4179 \\
\hline 17 & $\mathrm{I}(\mathrm{BH}-\mathrm{TH}) 3-1$ & 8.2706 & -0.4331 & 62 & I(VL-HT)325-1 & 9.4396 & 0.7359 \\
\hline
\end{tabular}




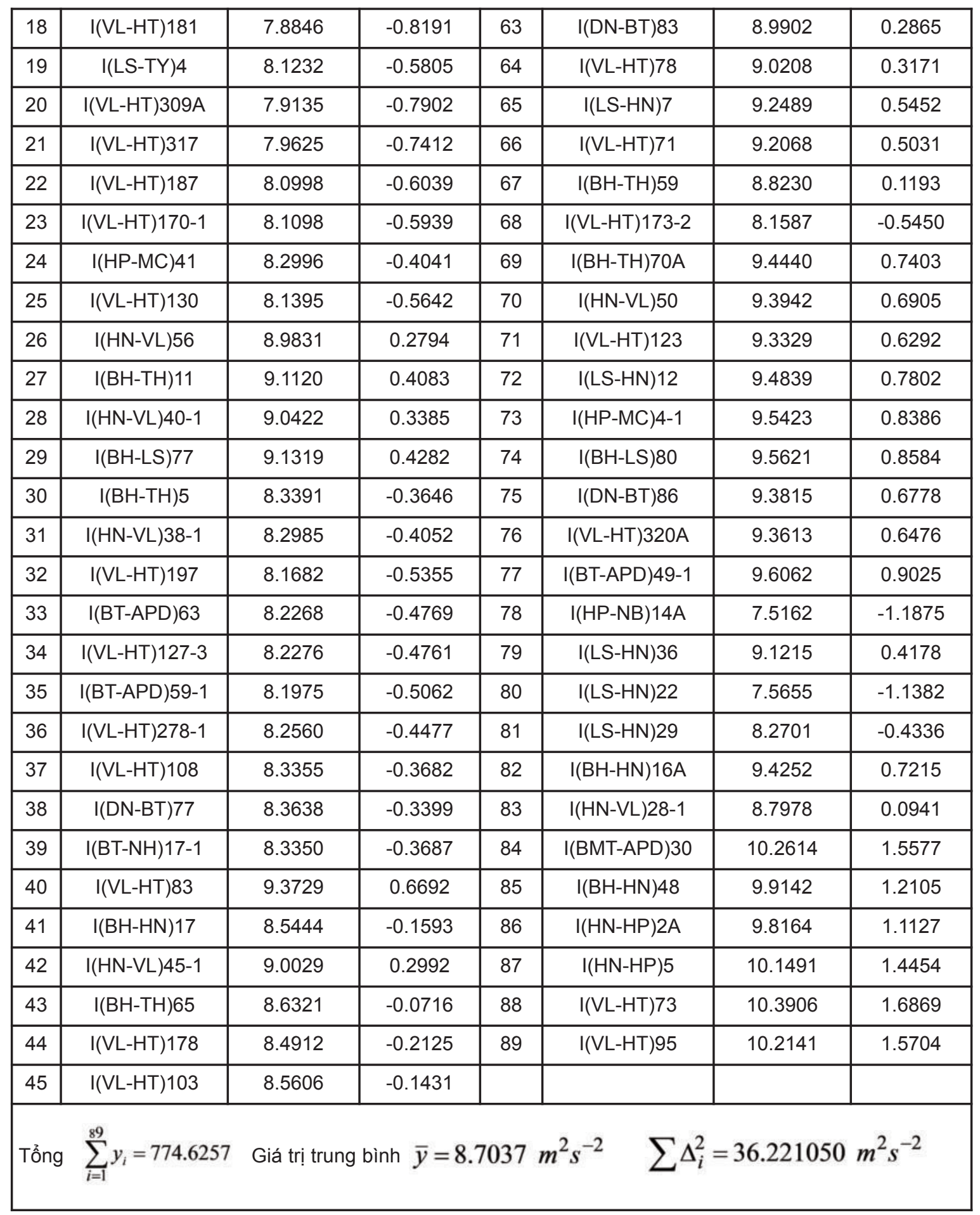


Bảng 3

\begin{tabular}{|c|c|c|c|}
\hline $\begin{array}{c}\text { STT } \\
\mathrm{i}\end{array}$ & Tên điểm & Trị đo $y_{i}$ & $\delta_{i}=y_{i}-\bar{y}$ \\
\hline 1 & I(DN-BT)16 & 7.7587 & -1.3011 \\
\hline 2 & $\mathrm{I}(\mathrm{VL}-\mathrm{HT}) 150$ & 7.7737 & -1.2861 \\
\hline 3 & $\mathrm{I}(\mathrm{HN}-\mathrm{VL}) 10 \mathrm{~A}$ & 7.8000 & -1.2598 \\
\hline 4 & I(DN-BT)28 & 7.8167 & -1.2431 \\
\hline 5 & $\mathrm{I}(\mathrm{VL}-\mathrm{HT}) 181$ & 7.8846 & -1.1752 \\
\hline 6 & I(VL-HT)309A & 7.9135 & -1.1463 \\
\hline 7 & I(VL-HT)317 & 7.9625 & -1.0973 \\
\hline 8 & $\mathrm{I}(\mathrm{HN}-\mathrm{VL}) 34-1$ & 8.0050 & -1.0548 \\
\hline 9 & I(HP-MC)48A & 8.0452 & -1.0146 \\
\hline 10 & I(VL-HT)187 & 8.0998 & -0.9600 \\
\hline 11 & $\mathrm{I}(\mathrm{VL}-\mathrm{HT}) 170-1$ & 8.1098 & -0.9500 \\
\hline 12 & I(LS-TY)4 & 8.1232 & -0.9366 \\
\hline 13 & $\mathrm{I}(\mathrm{VL}-\mathrm{HT}) 130$ & 8.1395 & -0.9203 \\
\hline 14 & $\mathrm{I}(\mathrm{VL}-\mathrm{HT}) 152-1$ & 8.2567 & -0.8031 \\
\hline 15 & $\mathrm{I}(\mathrm{BH}-\mathrm{TH}) 3-1$ & 8.2706 & -0.7892 \\
\hline 16 & I(HP-MC)41 & 8.2996 & -0.7602 \\
\hline 17 & $\mathrm{I}(\mathrm{BH}-\mathrm{TH}) 5$ & 8.3391 & -0.7207 \\
\hline 18 & $\mathrm{I}(\mathrm{BH}-\mathrm{TH}) 122 \mathrm{~A}$ & 8.5142 & -0.5456 \\
\hline 19 & $\mathrm{I}(\mathrm{BH}-\mathrm{HN}) 42$ & 8.5735 & -0.4863 \\
\hline 20 & $\mathrm{I}(\mathrm{HN}-\mathrm{VL}) 6-1$ & 8.6515 & -0.4083 \\
\hline 21 & $\mathrm{I}(\mathrm{BH}-\mathrm{HN}) 33$ & 8.7987 & -0.2611 \\
\hline 22 & $\mathrm{I}(\mathrm{BH}-\mathrm{HN}) 39$ & 8.8476 & -0.2122 \\
\hline 23 & $\mathrm{I}(\mathrm{HN}-\mathrm{VL}) 4-1$ & 8.9354 & -0.1244 \\
\hline 24 & $\mathrm{I}(\mathrm{BH}-\mathrm{TH}) 119$ & 8.9647 & -0.0951 \\
\hline 25 & I(HN-VL)56 & 8.9831 & -0.0767 \\
\hline 26 & $\mathrm{I}(\mathrm{HN}-\mathrm{VL}) 40-1$ & 9.0422 & -0.0176 \\
\hline 27 & $\mathrm{I}(\mathrm{BH}-\mathrm{TH}) 11$ & 9.1120 & 0.0522 \\
\hline 28 & $\mathrm{I}(\mathrm{BH}-\mathrm{LS}) 77$ & 9.1319 & 0.0721 \\
\hline 29 & $\mathrm{I}(\mathrm{HN}-\mathrm{HP}) 7$ & 9.2878 & 0.2280 \\
\hline 30 & $\mathrm{I}(\mathrm{LS}-\mathrm{HN}) 12$ & 9.4839 & 0.4241 \\
\hline
\end{tabular}


Nghiên cứu

\begin{tabular}{|c|c|c|c|}
\hline 31 & I(HP-MC)4-1 & 9.5423 & 0.4825 \\
\hline 32 & I(BH-LS)80 & 9.5621 & 0.5023 \\
\hline 33 & I(BT-APD)49-1 & 9.6062 & 0.5464 \\
\hline 34 & I(BH-LS)97 & 9.6207 & 0.5609 \\
\hline 35 & $\mathrm{I}(\mathrm{HN}-\mathrm{HP}) 2 \mathrm{~A}$ & 9.8164 & 0.7566 \\
\hline 36 & I(BH-HN)48 & 9.9142 & 0.8544 \\
\hline 37 & $\mathrm{I}(\mathrm{HN}-\mathrm{HP}) 5$ & 10.1491 & 1.0893 \\
\hline 38 & I(VL-HT)95 & 10.2141 & 1.1543 \\
\hline 39 & I(BMT-APD)30 & 10.2614 & 1.2016 \\
\hline \multirow[t]{3}{*}{40} & I(VL-HT)73 & 10.3906 & 1.3308 \\
\hline & Tổng & 362.3924 & $\sum \delta^{2}=27.6530092$ \\
\hline & Giá trị trung bình & 9.0598 & \\
\hline
\end{tabular}

Sai số trung phương:

$$
S=\sqrt{\frac{27.6530092}{40}}=0.8315 \mathrm{~m}^{2} \cdot \mathrm{s}^{-2} .
$$

$(\mathrm{y})_{\min }=7.7587 \mathrm{~m}^{2} \cdot \mathrm{s}^{-2} ; \mathrm{r}_{\min }=1.565 ;(\mathrm{y})_{\max }=10.3906 \mathrm{~m}^{2} \cdot \mathrm{s}^{-2} ; \mathrm{r}_{\max }=1.600$

Với $q=5 \%, n=40: \bar{r}=3.075$. Do cả hai giá trị $r_{\min }$ và $r_{\max }$ đều nhỏ hơn $\overline{\bar{r}^{3}}$, nên trong dãy các trị đo $y_{i}$ trong bảng 3 không có giá trị chứa sai số thô.

Bảng 4

\begin{tabular}{|c|c|c|c|}
\hline $\begin{array}{c}\text { STT } \\
\mathrm{i}\end{array}$ & Tên điểm & Trị đo $y_{i}$ & $\delta_{i}=y_{i}-\bar{y}$ \\
\hline 1 & I(HP-NB)14A & 7.5162 & -1.2745 \\
\hline 2 & $\mathrm{I}(\mathrm{LS}-\mathrm{HN}) 22$ & 7.5655 & -1.2252 \\
\hline 3 & $\mathrm{I}(\mathrm{VL}-\mathrm{HT}) 173-2$ & 8.1587 & -0.6320 \\
\hline 4 & $\mathrm{I}(\mathrm{VL}-\mathrm{HT}) 197$ & 8.1682 & -0.6225 \\
\hline 5 & $\mathrm{I}(\mathrm{BT}-\mathrm{APD}) 59-1$ & 8.1975 & -0.5932 \\
\hline 6 & $\mathrm{I}(\mathrm{BT}-\mathrm{APD}) 63$ & 8.2268 & -0.5639 \\
\hline 7 & $\mathrm{I}(\mathrm{VL}-\mathrm{HT}) 127-3$ & 8.2276 & -0.5631 \\
\hline 8 & $\mathrm{I}(\mathrm{VL}-\mathrm{HT}) 278-1$ & 8.2560 & -0.5347 \\
\hline 9 & $\mathrm{I}(\mathrm{LS}-\mathrm{HN}) 29$ & 8.2701 & -0.5206 \\
\hline
\end{tabular}


Nghiên cứu

\begin{tabular}{|c|c|c|c|}
\hline 10 & $\mathrm{I}(\mathrm{HN}-\mathrm{VL}) 38-1$ & 8.2985 & -0.4922 \\
\hline 11 & I(BT-NH)17-1 & 8.3350 & -0.4557 \\
\hline 12 & I(VL-HT)108 & 8.3355 & -0.4552 \\
\hline 13 & I(DN-BT)77 & 8.3638 & -0.4269 \\
\hline 14 & I(VL-HT)178 & 8.4912 & -0.2995 \\
\hline 15 & $\mathrm{I}(\mathrm{BH}-\mathrm{HN}) 17$ & 8.5444 & -0.2463 \\
\hline 16 & I(VL-HT)103 & 8.5606 & -0.2301 \\
\hline 17 & I(VL-HT)329A & 8.5690 & -0.2217 \\
\hline 18 & $\mathrm{I}(\mathrm{VL}-\mathrm{HT}) 141-3$ & 8.5696 & -0.2211 \\
\hline 19 & I(BH-TH)59 & 8.8230 & 0.0323 \\
\hline 20 & I(BH-LS)88-1 & 8.9457 & 0.1550 \\
\hline 21 & $\mathrm{I}(\mathrm{BH}-\mathrm{LS}) 93$ & 8.9653 & 0.1746 \\
\hline 22 & I(VL-HT)87 & 8.9815 & 0.1908 \\
\hline 23 & $\mathrm{I}(\mathrm{BH}-\mathrm{LS}) 85-1$ & 8.9849 & 0.1942 \\
\hline 24 & $\mathrm{I}(\mathrm{DN}-\mathrm{BT}) 83$ & 8.9902 & 0.1995 \\
\hline 25 & $\mathrm{I}(\mathrm{HN}-\mathrm{VL}) 45-1$ & 9.0029 & 0.2122 \\
\hline 26 & I(BH-LS)71 & 9.0143 & 0.2236 \\
\hline 27 & $\mathrm{I}(\mathrm{VL}-\mathrm{HT}) 78$ & 9.0208 & 0.2301 \\
\hline 28 & $\mathrm{I}(\mathrm{LS}-\mathrm{HN}) 36$ & 9.1215 & 0.3308 \\
\hline 29 & I(LS-TY)1 & 9.1216 & 0.3309 \\
\hline 30 & $\mathrm{I}(\mathrm{VL}-\mathrm{HT}) 71$ & 9.2068 & 0.4161 \\
\hline 31 & I(LS-HN)7 & 9.2489 & 0.4582 \\
\hline 32 & I(VL-HT)121 & 9.2843 & 0.4936 \\
\hline 33 & I(VL-HT)123 & 9.3329 & 0.5422 \\
\hline 34 & $\mathrm{I}(\mathrm{VL}-\mathrm{HT}) 320 \mathrm{~A}$ & 9.3613 & 0.5706 \\
\hline 35 & $\mathrm{I}(\mathrm{VL}-\mathrm{HT}) 83$ & 9.3729 & 0.5822 \\
\hline 36 & I(DN-BT)86 & 9.3815 & 0.5908 \\
\hline 37 & $\mathrm{I}(\mathrm{HN}-\mathrm{VL}) 50$ & 9.3942 & 0.6035 \\
\hline 38 & $\mathrm{I}(\mathrm{BH}-\mathrm{HN}) 16 \mathrm{~A}$ & 9.4252 & 0.6345 \\
\hline 39 & I(VL-HT)325-1 & 9.4396 & 0.6489 \\
\hline \multirow[t]{3}{*}{40} & $\mathrm{I}(\mathrm{BH}-\mathrm{TH}) 70 \mathrm{~A}$ & 9.4440 & 0.6533 \\
\hline & Tổng & 351.6265 & $\sum \delta^{2}=10.682331$ \\
\hline & Giá trị trung bình & 8.7907 & \\
\hline
\end{tabular}


Sai số trung phương:

$$
S=\sqrt{\frac{10.682331}{40}}=0.517 \mathrm{~m}^{2} \cdot \mathrm{s}^{-2}
$$

$(\mathrm{y})_{\min }=7.5162 \mathrm{~m}^{2} \cdot \mathrm{s}^{-2} ; \mathrm{r}_{\min }=2.465 ;(\mathrm{y})_{\max }=9.4440 \mathrm{~m}^{2} \cdot \mathrm{s}^{-2} ; \mathrm{r}_{\max }=1.264$

Với q $=5 \%, n=40: \bar{r}=3.075$. Do cả hai giá trị $r_{\min }$ và $r_{\text {max }}$ đều nhỏ hơn $\overline{\bar{x}}$, nên trong dãy các trị đo $y_{i}$ trong bảng 4 không có giá trị chứa sai số thô.

Bảng 5

\begin{tabular}{|c|c|c|c|}
\hline $\begin{array}{c}\text { STT } \\
\mathrm{i}\end{array}$ & Tên điểm & Trị đo $y_{i}$ & $\delta_{i}=y_{i}-\bar{y}$ \\
\hline 1 & $\mathrm{I}(\mathrm{BH}-\mathrm{TH}) 65$ & 8.6321 & -0.2521 \\
\hline 2 & $\mathrm{I}(\mathrm{HN}-\mathrm{VL}) 64$ & 8.6503 & -0.2339 \\
\hline 3 & $\mathrm{I}(\mathrm{VL}-\mathrm{HT}) 158$ & 8.7066 & -0.1776 \\
\hline 4 & $\mathrm{I}(\mathrm{HN}-\mathrm{VL}) 72$ & 8.7187 & -0.1655 \\
\hline 5 & $\mathrm{I}(\mathrm{VL}-\mathrm{HT}) 98$ & 8.7955 & -0.0887 \\
\hline 6 & $\mathrm{I}(\mathrm{HN}-\mathrm{VL}) 28-1$ & 8.7978 & -0.0864 \\
\hline 7 & $\mathrm{I}(\mathrm{BT}-\mathrm{APD}) 56$ & 8.8138 & -0.0704 \\
\hline 8 & $\mathrm{I}(\mathrm{DNN}-\mathrm{BT}) 74$ & 8.9212 & 0.0370 \\
\hline 9 & $\mathrm{I}(\mathrm{VL}-\mathrm{HT}) 247 \mathrm{~A}$ & 8.9213 & 0.0371 \\
\hline & Tổng & 79.9573 & $\sum \delta^{2}=0.200230$ \\
\hline & Giá trị trung bình $\bar{y}$ & 8.8842 & \\
\hline
\end{tabular}

$$
S=\sqrt{\frac{0.20230137578}{9}}=0.149 \mathrm{~m}^{2} \cdot \mathrm{s}^{-2} .
$$

$(\mathrm{y})_{\min }=8.6321 \mathrm{~m}^{2} \cdot \mathrm{s}^{-2} ; \mathrm{r}_{\min }=1.692 ;(\mathrm{y})_{\max }=8.9213 \mathrm{~m}^{2} \cdot \mathrm{s}^{-2} ; \mathrm{r}_{\max }=0.249$

Với q $=5 \%, \mathrm{n}=9: \bar{r}=2.349$. Do cả hai giá trị $\mathrm{r}_{\min }$ và $\mathrm{r}_{\text {max }}$ đều nhỏ hơn $\overline{\bar{x}}$, nên trong dãy các trị đo $y_{i}$ trong bảng 5 không có giá trị chứa sai số thô.

Như vậy trong dãy bao gồm 89 các trị đo $y_{i}$ ở bảng 2 không chứa các trị đo thô. Từ các kết quả tính toán giá trị trung bình $\bar{\gamma}$ từ 89 các trị đo trong bảng 2 , chúng ta nhận được $\bar{y}=8.7037 \mathrm{~m}^{2} \mathrm{~s}^{-2}$. Từ công thức (6) chúng ta nhận được giá trị thế trọng trường $\mathrm{W}_{\mathrm{o}}=$ $62636847.296 \mathrm{~m}^{2} . \mathrm{s}^{-2}$ của mặt geoid cục bộ Hòn Dấu. So với giá trị thế trọng trường chính thức $\mathrm{W}_{\mathrm{O}}=62636847.291 \mathrm{~m}^{2} \cdot \mathrm{s}^{-2}$, khi nhận giá trị trung bình của gia tốc lực trọng trường chuẩn trên lãnh thổ Việt Nam $\bar{\gamma}=9.785 \mathrm{~ms}^{-2}$, độ chênh $0.005 \mathrm{~m}^{2} . \mathrm{s}^{-2}$ của các giá trị thế trọng trường $W_{0}$ chỉ tương ứng với sự xê dịch của các mặt đẳng thế cục bộ tại trạm nghiệm triều 
Hòn Dấu ở mức nhỏ bỏ qua $\frac{0.005 \mathrm{~m}^{2} \cdot \mathrm{s}^{-2}}{9.785 \mathrm{~m}_{\mathrm{s}} \mathrm{s}^{-2}}=0.5 \mathrm{~mm}$. Điều này một lần nữa xác định sự đúng

đắn của giá trị thế trọng trường chính thức $\mathrm{W}_{0}=62636847.291 \mathrm{~m}^{2} \cdot \mathrm{s}^{-2}$.

Bây giờ chúng ta kiểm tra độ cao $H_{0}=\frac{\bar{W}_{0}-W_{0}}{\bar{\gamma}_{M}}$ của mặt geoid cục bộ Hòn Dấu so với mặt geoid toàn cầu dựa trên mô hình địa hình động lực trung bình DTU10MDT tại trạm nghiệm triều Hòn Dấu. Mô hình DTU10MDT do Trung tâm Vũ trụ quốc gia Đan Mạch (DNSC) thuộc Trường Đại học Tổng hợp kỹ thuật Đan Mạch (DTU) thành lập trên toàn bộ các biển và các đại dương thế giới dựa trên các dữ liệu altimetry từ các dự án vệ tinh TOPEX/POSEIDON, JASON-1, GEOSAT, GFO, ERS-1, ERS-2, ICESAT, ENVISAT trong giai đoạn từ 1993 - 2004. Giá trị MDT được xác định tại một vị trí trên các biển và các đại dương thế giới (trong hệ không phụ thuộc triều) từ mô hình DTU10MDT chính là độ cao của mặt biển trung bình tại vị trí đó so với mặt geoid toàn cầu $\bar{W}_{0}=62636856.00 \mathrm{~m}^{2} . \mathrm{s}^{-2}$. Tại trạm nghiệm triều Hòn Dấu với các tọa độ trắc địa trong hệ WGS84 $\mathrm{B}=20^{\circ} 40^{\prime} 06^{\prime \prime} .626$, $\mathrm{L}=106^{\circ} 48^{\prime} 52^{\prime \prime} .208$, chúng ta xác định giá trị MDT từ mô hình DTU10MDT và chuyển giá trị này từ hệ không phụ thuộc triều về hệ triều 0 . Các kết quả tính toán được trình bày ở bảng 6 ở dưới đây.

Bảng 6

\begin{tabular}{|c|c|c|c|}
\hline Tên trạm & $\begin{array}{c}\text { Độ cao của mặt biển trung } \\
\text { bình toàn cầu so với mặt } \\
\text { geoid toàn cầu } \overline{M D T}_{n}(\mathrm{~m})\end{array}$ & $\begin{array}{c}\text { Số hiệu chỉnh chuyển từ hệ } \\
\text { không phụ thuộc triều về hệ } \\
\text { triều } 0(\mathrm{~m})\end{array}$ & $\begin{array}{c}\text { Giá trị } \overline{M D T}_{z} \text { tương } \\
\text { ứng geoid toàn cầu } \\
\text { trong hệ triều } 0(\mathrm{~m})\end{array}$ \\
\hline Hòn Dấu & 0.874 & 0.021 & 0.895 \\
\hline
\end{tabular}

Giá trị độ cao $\mathrm{H}_{\mathrm{o}}=0.895 \mathrm{~m}$ được tính từ mô hình DTU10MDT chỉ chênh với giá trị chính thức $\mathrm{H}_{\mathrm{o}}=0.890 \mathrm{~m}$ có $5 \mathrm{~mm}$ nhỏ bỏ qua. Việc tính toán từ mô hình DTU10MDT cho giá trị $\mathrm{H}_{\mathrm{o}}$ một cách khách quan, không phụ thuộc vào trạng thái của các điểm độ cao hạng I quốc gia cũng như các kết quả đo GPS trên các điểm này.

\section{Kết luận}

Các kết quả tính toán kiểm tra giá trị thế trọng trường $\mathrm{W}_{0}=62636847.291 \mathrm{~m}^{2} \cdot \mathrm{s}^{-2}$ và giá trị độ cao $\mathrm{H}_{\mathrm{o}}=0.890 \mathrm{~m}$ của mặt geoid cục bộ Hòn Dấu so với mặt geoid toàn cầu dựa trên 89 điểm độ cao hạng I và mô hình DTU10MDT một lần nữa khẳng định sự đúng đắn của các giá trị nêu trên. Trong bài báo khoa học này cũng đã luận giải sự không đổi của giá trị $\mathrm{H}_{\mathrm{o}}=0.890 \mathrm{~m}$ trên toàn bộ lãnh thổ Việt Nam. Các giá trị nêu trên là các giá trị cơ sở để giải quyết nhiều bài toán khoa học - kỹ thuật hiện đại của trắc địa vật lý ở nước ta.

Các tác giả cám ơn Chương trình KH\&CN trọng điểm cấp Nhà nước KC-09/11-15 "Nghiên cứu Khoa học và Công nghệ phục vụ quản lý biển, hải đảo và phát triển kinh tế biển" đã hỗ trợ nội dung bài báo này. $\mathrm{O}$

\section{Tài liệu tham khảo}

[1]. Bursa M., Kenyon S., Kouba J., Radj K., Vatrt V., Vojtiskov, Simek J. (2002). Word 
Height System specified by geopotential at tide gauge stations. IAG Symposia 124, 291296. Springer

[2]. Bursa M., Kenyon S., Kouba J., Sima Z., Vatrt V., Vitek V. and Vojtiskova M. (2007). The geopotential value $W 0$ for specifying the relativistic atomic time scale and a global vertical reference system. J. of Geodesy, 81, 2, pp. 103-110.

[3]. Dennis D. McCarthy, Gerard Petit. (2004). IERS Conventions (2003). IERS Technical Note No 32. Frankfurt am Main, 2004.

[4]. Hà Minh Hòa (2007). Giải quyết một số vấn đề liên quan đến việc chuyển hệ độ cao được xác định từ mặt nước biển trung bình ở trạm thủy triều về mặt Quasigeoid toàn cầu. Tạp chí Địa chính số 2, tháng 4/2007, trg. 3 - 11.

[5]. Hà Minh Hòa, Nguyễn Thị Thanh Hương và nnk (2012). Nghiên cứu cơ sở khoa học của việc hoàn thiện hệ độ cao gắn liền với việc xây dựng hệ tọa độ động lực quốc gia. Đề tài khoa học và công nghệ cấp Bộ Tài nguyên và Môi trường giai đoạn 2010 - 2012. Hà Nội - 2012.

[6]. Ha Minh Hoa (2013). Estimating the geopotential value $W_{0}$ of the local geoid based on data from local and global normal heights of GPS/Leveling points in Vietnam. Geodesy and Cartography. Taylor \& Francis. UDK 528.21, doi:10.3846/20296991.2013.823705, V.39 (3): 99-105.

[7]. Hà Minh Hòa (2014). Lý thuyết và thực tiễn của Trọng lực trắc địa. NXB Khoa học và Kỹ thuật, 592 trg., Hà Nội - 2014.

[8]. Nguyễn Tuấn Anh (2015). Nghiên cứu chi tiết độ cao của mặt geoid cục bộ Hòn Dấu so với mặt geoid toàn cầu trên lãnh thổ Việt Nam. Tạp chí Khoa học Đo đạc và Bản đồ, No25, 09/2015.

[9]. Petit G., Luzum B. (2010). IERS Conventions (2010). IERS Technical Note No 36, Verlag dés Bundesamts fur Kartographie und Geodasie. Frankfurt am Main 2010, 179 pp.

[10]. Tenzer R., Vatrt V. and Amos M. (2009). Realization of the World Height System in New Zealand: Preliminary Study, pp. 343 - 349. Geodesy for Planet Earth, Proceedings of the 2009 IAG Symposium, Buenos Aires, Argentina, 31 August - 4 September 2009. International Association of Geodesy Symposia, Vol. 136, 2012, Steve Kenyon. O

\section{Summary}

Assoc. Prof. Dr. Sc. Ha Minh Hoa, MSc. Nguyen Thi Thanh Huong

Vietnam Institute of Geodesy and Cartography

MSc. Luong Thanh Thach, Hanoi University of Natural Resources and Environment

\section{Checking estimation of the geopotential $\mathrm{W}_{\mathrm{o}}$ of the local geoid Hon Dau based on 89 first order benchmarks}

This scientific article accomplished checking calculation of the geopotential $\mathrm{W}_{\mathrm{O}}=$ $62636847.291 \mathrm{~m}^{2} . \mathrm{s}^{-2}$ and height $\mathrm{H}_{\mathrm{o}}=0.890 \mathrm{~m}$ of the hon Dau local geoid corresponding with the global geoid in relation based on 89 first order benchmarks and international model of DTU10MDT. Checking results more show the confidence of abovementioned values and makes base on their using for solving modern tasks of physical geodesy in our country. $O$ 